\title{
Cyclical changes in seroprevalence of leptospirosis in California sea lions: endemic and epidemic disease in one host species?
}

\author{
James O Lloyd-Smith*1, Denise J Greig'2, Sharon Hietala ${ }^{3}$, George S Ghneim 4 , \\ Lauren Palmer ${ }^{5}$, Judy St Leger ${ }^{6}$, Bryan T Grenfell ${ }^{1}$ and Frances MD Gulland ${ }^{2}$
}

\begin{abstract}
Address: ${ }^{1}$ Center for Infectious Disease Dynamics, Pennsylvania State University, University Park, USA, ${ }^{2}$ The Marine Mammal Center, Marin Headlands, 1065 Fort Cronkhite, Sausalito, USA, ${ }^{3}$ California Animal Health and Food Safety Laboratory, University of California, Davis, USA ${ }^{4}$ Research Triangle Institute International, Research Triangle Park, USA, ${ }^{5}$ Marine Mammal Care Center, Fort Macarthur, San Pedro, USA and ${ }^{6}$ SeaWorld of California, San Diego, USA

Email: James O Lloyd-Smith* - jlloydsmith@psu.edu; Denise J Greig - greigd@tmmc.org; Sharon Hietala - skhietala@ucdavis.edu; George S Ghneim - George.Ghneim@ncmail.net; Lauren Palmer - lpalmer333@aol.com; Judy St Leger - Judy.St.Leger@seaworld.com; Bryan T Grenfell - grenfell@psu.edu; Frances MD Gulland - gullandf@tmmc.org

* Corresponding author
\end{abstract}

Published: 6 November 2007

BMC Infectious Diseases 2007, 7:125 doi:10.1186/147/-2334-7-125
Received: 23 February 2007

Accepted: 6 November 2007

This article is available from: http://www.biomedcentral.com/I47I-2334/7/I25

(C) 2007 Lloyd-Smith et al; licensee BioMed Central Ltd.

This is an Open Access article distributed under the terms of the Creative Commons Attribution License (http://creativecommons.org/licenses/by/2.0), which permits unrestricted use, distribution, and reproduction in any medium, provided the original work is properly cited.

\begin{abstract}
Background: Leptospirosis is a zoonotic disease infecting a broad range of mammalian hosts, and is re-emerging globally. California sea lions (Zalophus californianus) have experienced recurrent outbreaks of leptospirosis since 1970, but it is unknown whether the pathogen persists in the sea lion population or is introduced repeatedly from external reservoirs.
\end{abstract}

Methods: We analyzed serum samples collected over an I I-year period from I 344 California sea lions that stranded alive on the California coast, using the microscopic agglutination test (MAT) for antibodies to Leptospira interrogans serovar Pomona. We evaluated seroprevalence among yearlings as a measure of incidence in the population, and characterized antibody persistence times based on temporal changes in the distribution of titer scores. We conducted multinomial logistic regression to determine individual risk factors for seropositivity with high and low titers.

Results: The serosurvey revealed cyclical patterns in seroprevalence to $L$. interrogans serovar Pomona, with 4-5 year periodicity and peak seroprevalence above $50 \%$. Seroprevalence in yearling sea lions was an accurate index of exposure among all age classses, and indicated on-going exposure to leptospires in non-outbreak years. Analysis of titer decay rates showed that some individuals probably maintain high titers for more than a year following exposure.

Conclusion: This study presents results of an unprecedented long-term serosurveillance program in marine mammals. Our results suggest that leptospirosis is endemic in California sea lions, but also causes periodic epidemics of acute disease. The findings call into question the classical dichotomy between maintenance hosts of leptospirosis, which experience chronic but largely asymptomatic infections, and accidental hosts, which suffer acute illness or death as a result of disease spillover from reservoir species. 


\section{Background}

Leptospirosis is an acute febrile zoonosis of global importance, caused by spirochetes of the genus Leptospira [1-5]. Owing to recent epidemics in humans in Nicaragua [6], Brazil [7], India [8] and South East Asia [9], and rising incidence in domestic dogs $[10,11]$, leptospirosis has been identified as an emerging infectious disease $[4,12]$. Outbreaks in humans are usually seasonal and associated with flooding or activities involving exposure to contaminated water or animal tissues, such as swimming, hunting, farming, and working in abattoirs or veterinary settings. Designing public health measures to control leptospirosis requires an understanding of the ecology of the disease in its many wild and domestic mammalian hosts. The Leptospira interrogans complex (sensu lato) includes $>200$ pathogenic serovars [2], which differ widely in their interactions with different host species. A central tenet of the epidemiology of leptospirosis is the distinction between maintenance hosts and accidental hosts for a given serovar, or, equivalently, between host-adapted and non-host-adapted serovars [1-3]. In this framework, maintenance hosts develop a chronic, largely asymptomatic infection of their proximal renal tubules, and may shed leptospires in their urine for months or years. In contrast, accidental hosts experience acute infections, with symptoms ranging from malaise to multi-organ failure and death.

In California, leptospirosis has resurged in humans and domestic dogs $[13,14]$. Disease outbreaks have also occurred in California sea lions (Zalophus californianus) off the central and northern coasts of California, with hundreds of animals dying in each outbreak. The first leptospirosis epidemic documented in California sea lions was in 1970, and Leptospira interrogans serovar Pomona was isolated from infected sea lions $[15,16]$. Since then there have been repeated epidemics, with an outbreak every three to five years since 1984 [17-19]. The epidemiology of the disease in sea lions is unclear, and the role of sea lions in maintaining this zoonotic disease is unknown. Crucially, it is unknown whether the recurring epidemics of leptospirosis in sea lions result from repeated introduction of the pathogen from external reservoirs, or from cyclic dynamics of an endemic disease. Numerous factors can cause epidemics to cycle, including environmental drivers, changes in host population density, antigenic changes by the pathogen, or changes in the proportion of the host population that is immune, associated with the concept of "herd immunity" [20]. The premise of herd immunity is that an epidemic cannot occur if a certain threshold proportion of a population is immune due to previous infection or vaccination (thus indirectly protecting susceptible individuals against infection). As the pool of susceptible individuals is replenished by birth or immigration, eventually an epidemic becomes possible again.

As the first step to elucidate the epidemiology of recurring leptospirosis outbreaks in California sea lions, and to identify future research directions, this study investigated changes in seroprevalence in an unusually detailed longitudinal dataset from 1995-2005. In an unvaccinated population, seroprevalence is a measure of past exposure to leptospirosis. However, the duration of seropositivity following leptospiral infection is not well known for any host $[1,21-23]$, and is completely unknown for sea lions. Because this is a crucial quantity for interpretation of serological time series, we analyzed available data to characterize the decay rate of antibody titers to leptospires.

\section{Methods \\ Sampling}

Serum samples were collected from California sea lions stranding along the central and northern California coast $\left(37^{\circ} 42^{\prime} \mathrm{N}, 123^{\circ} 05^{\prime} \mathrm{W}\right.$ to $\left.35^{\circ} 59^{\prime} \mathrm{N}, 121^{\circ} 30^{\prime} \mathrm{W}\right)$ and archived at The Marine Mammal Center (TMMC) at $70^{\circ} \mathrm{C}$. All animals were sampled during routine veterinary care while in rehabilitation as authorized by the National Marine Fisheries Service Research and Enhancement Permit to Take Marine Mammals (\# 932-1489-08), and approved by The Marine Mammal Center's Internal Animal Care and Use Committee. For each sex and age class, 20 samples for each year were randomly selected from the archive; when fewer than 20 samples were available for a given sex/age class and year, all samples were used (Table 1 ). Animals were classified as having stranded because of leptospirosis if serum chemistry results were indicative of leptospirosis (blood urea nitrogen $>100 \mathrm{mg} / \mathrm{dl}$, creatinine $>2 \mathrm{mg} / \mathrm{dl}$, sodium $>155 \mathrm{meq} / \mathrm{L}$ and phosphorus $>$ calcium) or if gross necropsy (swollen kidneys with loss of renule differentiation and pale tan renule cortices) and histopathology (interstitial nephritis) revealed renal disease consistent with leptospirosis [19]. To reduce potential bias arising from sampling only stranded sea lions, a reduced dataset was generated which excluded leptospirosis-induced strands.

Further serum samples from stranded California sea lions were obtained from two other stranding centers in California, SeaWorld in San Diego, CA (collection range $33^{\circ} 45^{\prime} \mathrm{N}, 118^{\circ} 07^{\prime} \mathrm{W}$ to $32^{\circ} 32^{\prime} \mathrm{N}, 117^{\circ} 07^{\prime} \mathrm{W}$ ) and the Marine Mammal Care Center at Fort Macarthur, CA (collection range $34^{\circ} 17^{\prime} \mathrm{N}, 119^{\circ} 30^{\prime} \mathrm{W}$ to $33^{\circ} 45^{\prime} \mathrm{N}$, $118^{\circ} 07^{\prime} \mathrm{W}$ ). All available samples were analyzed (Table 2).

\section{Serology}

Serum samples were submitted to the California Animal Health and Food Safety Laboratory (Davis, CA), and 
Table I: Sample sizes for TMMC seroprevalence time series, by sex and age. Total numbers and numbers excluding leptospirosisinduced strands (in parentheses) are shown

\begin{tabular}{|c|c|c|c|c|c|c|c|c|c|c|c|c|}
\hline Sex & Age & 1995 & 1996 & 1997 & 1998 & 1999 & 2000 & 2001 & 2002 & 2003 & 2004 & 2005 \\
\hline \multirow[t]{6}{*}{ Female } & Yearling & 8 & 16 & 19 & 23 & 8 & 18 & 21 & 19 & 38 & 20 & 29 \\
\hline & $(\mathrm{I}-2 \text { y.o. })^{\mathrm{a}}$ & (3) & $(14)$ & $(17)$ & $(22)$ & (7) & (II) & $(20)$ & $(18)$ & $(38)$ & (9) & (29) \\
\hline & Subadult & 9 & 6 & 19 & 23 & 11 & 19 & 16 & 12 & 20 & 13 & 5 \\
\hline & (2-5 у.о.) & (3) & (5) & (13) & (19) & $(2)$ & (13) & (II) & $(10)$ & $(16)$ & (7) & (5) \\
\hline & Adult & 9 & 7 & 23 & 19 & 18 & 20 & 20 & 18 & 20 & 19 & 20 \\
\hline & ( $\geq 5$ y.o.) & (5) & (5) & $(16)$ & $(16)$ & $(13)$ & (19) & $(18)$ & (17) & $(20)$ & (16) & (19) \\
\hline \multirow[t]{8}{*}{ Male } & Yearling & 12 & 18 & 19 & 18 & 17 & 17 & 18 & 20 & 38 & 18 & 36 \\
\hline & $(1-2 \text { y.o. })^{a}$ & (9) & $(18)$ & $(15)$ & $(18)$ & $(10)$ & $(12)$ & $(18)$ & (18) & (38) & (6) & (33) \\
\hline & Juvenile & 21 & 25 & 20 & 20 & 20 & 20 & 19 & 20 & 20 & 19 & 16 \\
\hline & (2-4 у.о.) & $(2)$ & $(16)$ & $(12)$ & (19) & (3) & (5) & (8) & (9) & $(12)$ & (2) & (II) \\
\hline & Subadult & 19 & 14 & 15 & 20 & 20 & 19 & 13 & 10 & 10 & 19 & 19 \\
\hline & (4-8 y.o.) & (3) & $(10)$ & (9) & (14) & (4) & (8) & $(10)$ & (6) & (9) & (9) & (9) \\
\hline & Adult & 12 & 8 & 9 & 4 & 5 & 8 & 14 & 9 & 8 & 17 & 6 \\
\hline & ( $\geq 8$ у.о.) & (7) & (8) & (7) & (3) & (4) & (7) & $(12)$ & (6) & (8) & (9) & (6) \\
\hline \multirow[t]{2}{*}{ Total } & & 90 & 94 & 124 & 127 & 99 & 121 & 121 & 108 & 154 & 125 & 131 \\
\hline & & $(32)$ & $(76)$ & (89) & $(\mathrm{III})$ & $(43)$ & (75) & $(97)$ & (84) & $(14 I)$ & (58) & $(112)$ \\
\hline
\end{tabular}

a The yearling class may include some pups 8-12 months of age, stranding in February to May.

assayed for Leptospira antibodies using the microscopic agglutination test (MAT) [24-26]. The endpoint reading of the microagglutination reaction was reported as the serum dilution at which $50 \%$ of the leptospires were agglutinated by direct observation using inverted field microscopy. Leptospira cultures and serovar-specific control sera were obtained from the USDA National Veterinary Services Laboratories (Ames, Iowa). A previous study evaluated the MAT for sera from California sea lions and reported $100 \%$ sensitivity at $\geq 1: 3200$ based on 19 positive controls (established by clinical signs of disease, lesions at necropsy, and visible leptospires in silver stained kidney sections) and 100\% specificity at $<1: 100$ based on 19 negative controls (captive-bred animals that had never exhibited signs of renal disease) [27].

Of the 1344 samples of the 1995-2005 time series, 724 were diluted to a maximum dilution of $1: 204800,17$ to a maximum dilution of 1:3200, and 553 to a maximum of 1:800. 50 samples were removed from the analysis due to contamination or incomplete data. The serum samples were assayed against six Leptospira serovars, representing the serovars of diagnostic interest in California during the study period: L. interrogans serovar Pomona, L. interrogans serovar Bratislava, L. kirschneri serovar Grippotyphosa, L. interrogans serogroup Icterohaemorrhagiae serovar Copenhageni, L. interrogans serovar Canicola, and L. interrogans serovar Hardjo type Hardjoprajitno. Most analyses reported here are based on the titer to serovar Pomona, which has been the serovar of all Leptospira isolates from wild California sea lions $[15,18,28]$. Samples that agglutinated at the 1:100 dilution were classified as seropositive [2,27], and we further distinguished between high-titer seropositives that agglutinated at dilutions of 1:800 or higher [23] and low-titer seropositives that agglutinated only at dilutions below 1:800.

\section{Analysis}

Graphical and statistical analyses were conducted using Matlab v6.1 (The Mathworks, Cambridge MA) and R [29]. Seroprevalences were calculated as binomial proportions with exact confidence intervals [30]. To determine individual risk factors, multinomial logistic regression [31,32] was conducted treating the serologic result (negative,

Table 2: Sample sizes for SeaWorld and Marine Mammal Care Center

\begin{tabular}{lccccccc}
\hline Source & Subset & 2000 & 2001 & 2002 & 2003 & 2004 & 2005 \\
\hline \multirow{2}{*}{ SeaWorld } & Total & 4 & 17 & 15 & 58 & 38 & 49 \\
\multirow{3}{*}{ MMCC } & Yearling & 0 & 4 & 1 & 17 & 14 & 14 \\
& Total & 0 & 0 & 0 & 0 & 40 & 12 \\
\hline
\end{tabular}


high-titer, low-titer) as a nominal outcome with the following covariates: age, sex, outbreak year $(1995,1999$, $2000,2004)$ versus non-outbreak year, and season (August-December versus January-July). These seasonal ranges were chosen to minimize the residual deviance of the regression. Outcomes of the multinomial logistic regression are assessed in terms of relative risk ratios (RRR), which describe the ratio between the relative risk of a given outcome versus a reference outcome (e.g. hightiter seropositivity versus seronegativity) for one factor compared to another (e.g. for male versus female individuals). Antibody half-life was estimated as the reciprocal of the slope of the number of two-fold decreases in titer versus time. Throughout the study, proportions were compared using a chi-squared test with continuity correction, or Fisher's exact test when the expected number in any category was $<5$ [33]. All statistical tests were two-tailed.

Yearling seroprevalence was assessed as an index of leptospirosis exposure by testing for linear relationship with the relative change (i.e. ratio of successive values) in hightiter seroprevalence for the whole population. (The mean of two seroprevalence estimates, derived from the full dataset and from the reduced dataset without leptospirosis strandings, was taken to be the most unbiased estimate of population seroprevalence; qualitatively similar results were obtained using either estimate on its own.) Possible non-linear effects were tested by performing a regression with a quadratic term, and normality of the residuals was assessed using a Kolmogorov-Smirnov test. The intercept of the linear regression, $b$, gives the relative change in high-titer seroprevalence when yearling seroprevalence is zero. If zero yearling seroprevalence indicates no new exposure for the population, then we can calculate the annual per capita probability that antibody decay will cause loss of high-titer status, $p_{\text {decar }}$. Let $N_{H T}$ and $N$ be the number of high-titer individuals and total population size, respectively, such that high-titer seroprevalence is $N_{H T} / N$. Let $p_{\text {death }}$ be the annual per capita probability of death, and $\lambda$ be the annual growth rate of the population such that $N(t)=\lambda N(t-1)$. If death and antibody decay are independent processes, and there is no new exposure in year $t$, then $N_{H T}(t)=\left(1-p_{\text {death }}\right)\left(1-p_{\text {decay }}\right) N_{H T}(t-1)$. If new recruits to the population are seronegative, as observed for sea lion pups [27], then:

$$
\begin{aligned}
b & =\frac{N_{H T}(t) / N(t)}{N_{H T}(t-1) / N(t-1)} \\
& =\frac{\left[\left(1-p_{\text {death }}\right)\left(1-p_{\text {decay }}\right) N_{H T}(t-1)\right] /[\lambda N(t-1)]}{N_{H T}(t-1) / N(t-1)} \\
& =\frac{\left(1-p_{\text {death }}\right)\left(1-p_{\text {decay }}\right)}{\lambda}
\end{aligned}
$$

and therefore

$$
p_{\text {decay }}=1-b \lambda /\left(1-p_{\text {death }}\right)
$$

Given approximate values $p_{\text {death }}=0.07$ and $\lambda=1.05$ [34], $p_{\text {decay }} \approx 1-1.13 b$.

\section{Results}

\section{Comparison of serovars}

To determine the dominant serovar(s) in this dataset, we tabulated which serovar(s) contributed the maximum titer score for each sample with one or more titer $\geq 1: 800$ (Table 3). Of 449 samples with at least one titer $\geq 1: 800$, serovar Pomona was the unique maximum for 201 (45\%) samples and was positive at the highest dilution measured for an additional 226 (50\%) samples. In only 19 of 449 (4\%) samples did another serovar have a higher titer than serovar Pomona. Qualitatively similar results were found for all samples with one or more titers $\geq 1: 100$. Because of the consistently higher titer scores for serovar Pomona, combined with the fact that all Leptospira isolates from wild California sea lions have been serovar Pomona $[15,18,28]$, we restrict the remainder of our analysis to serovar Pomona and attribute the positive titers to other serovars to the known cross-reactivity of the MAT $[10,35]$.

\section{Seroprevalence time series, 1995-2005}

Seroprevalence of leptospiral antibodies in 1338 stranded California sea lions exhibited cycles of $4-5$ year periodicity over the duration of the study (Figure 1a). Peaks in

\begin{tabular}{|c|c|c|c|c|c|c|c|}
\hline Serovar & Pomona & Bratislava & Canicola & Grippo. & Hardjo & Ictero. & Total samples \\
\hline Unique maximuma & 201 & 9 & 3 & 2 & 2 & 1 & 218 \\
\hline Shared maximum (detection limit) ${ }^{b}$ & 226 & 212 & 100 & 114 & 79 & 132 & 226 \\
\hline Shared maximum (below limit)c & 3 & 3 & 0 & 2 & 1 & 2 & 5 \\
\hline Titer $\geq 1: 800^{d}$ & 435 & 391 & 221 & 271 & 217 & 302 & 449 \\
\hline
\end{tabular}
seroprevalence corresponded to reported outbreaks of leptospirosis in 1995, 1999, 2000, and 2004. Cycles were

Table 3: Summary of serovars corresponding to maximum titer scores for all samples with at least one titer $\geq 1: 800$

a Samples for which only one serovar had the maximum score.

b Samples for which two or more serovars shared the maximum score, which was the highest dilution measured for that sample.

c Samples for which two or more serovars shared the maximum score, at a titer below the highest dilution measured for that sample.

d Number of samples with titers $\geq 1: 800$ for each serovar. 
most evident in seroprevalence estimates derived from all stranded sea lions (solid lines); when leptospirosisinduced strands were removed from the analysis (dashed lines), the cyclic pattern remained but the 2004 peak was greatly reduced. The cyclic variation arose in the prevalence of high antibody titers $(\geq 1: 800)$ reflective of recent exposure, while the proportion of individuals with lower positive titers was constant within uncertainties (Figure $1 b, c)$.

High-titer seroprevalence remains at $10-30 \%$ in non-outbreak years, possibly indicating on-going transmission of leptospirosis. We explored the use of yearling seroprevalence as an index of incidence of leptospirosis in a given year. Sea lion pups remain on the rookery islands for roughly one year after birth [36] and do not appear to be exposed on the rookeries (unpublished data), so yearlings are in their first year of possible exposure to leptospirosis. Yearling seroprevalence exhibited strong peaks in outbreak years, but in intervening years dropped to lower levels than the population seroprevalence (Figure 2a). Seropositive yearlings were observed in all years except 1996 (0/33, 95\% CI for binomial proportion: 0-0.11), indicating that exposure to $L$. interrogans serovar Pomona continued at low levels between outbreaks. Yearling seroprevalence was strongly correlated with exposure levels for all ages, as measured by the relative year-to-year change in high-titer seroprevalence $\left(y=0.28+4.3 x, R^{2}=\right.$ 0.80 ; Figure $2 \mathrm{~b}$ ). (When the dependent variable was based on seroprevalence estimates with yearlings excluded to avoid possible circularity, the correlation was even stronger $\left(R^{2}=0.88\right)$, but the whole-population results are shown because they inform our work on antibody decay, below.) In 2000, the overall increase in seroprevalence was small compared to a yearling seroprevalence $>50 \%$, because of a large number of strandings caused by domoic acid toxicity in that year, which diluted the influence of animals stranding because of leptospirosis [19]. When the point for year 2000 was excluded as an outlier, the linear relationship was much stronger $\left(y=0.17+5.7 x, R^{2}=0.98\right.$; Figure $2 \mathrm{~b}$ ). For both regressions, a quadratic term was not supported and residuals did not deviate significantly from normality.

\section{Individual risk factors and asymptomatic seropositives}

Multinomial logistic regression was performed to assess individual risk factors for high-titer and low-titer seropositivity (Table 4). The relative risk of high titer versus seronegativity was 7.0 times higher in outbreak years than in non-outbreak years, and 4.9 times higher during the August-December season when most leptospirosis strands are reported. The relative risk of high titers was 4.1 -fold higher for males than for females. The relative risk of high titers is highest for juveniles and subadults, and drops (a)

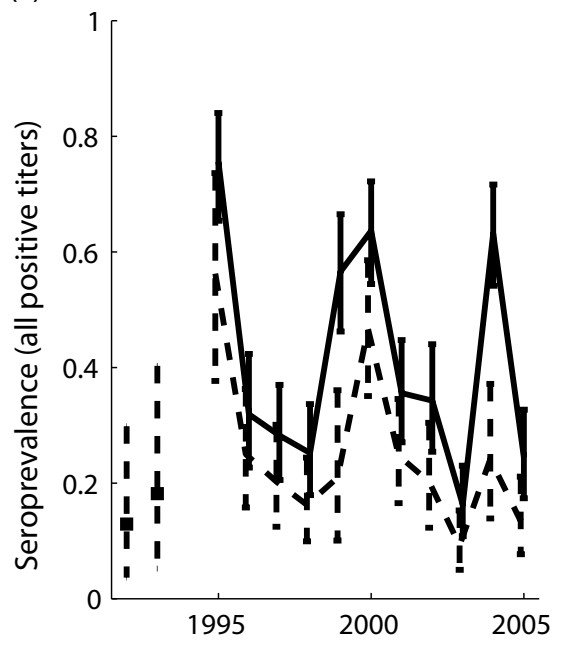

(b)

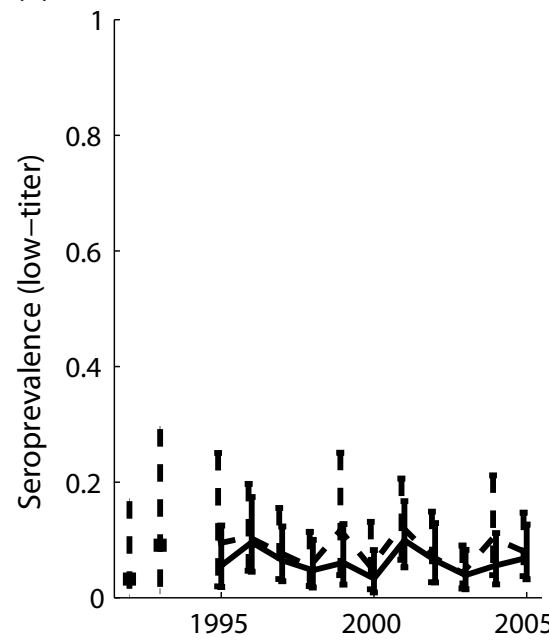

(c)

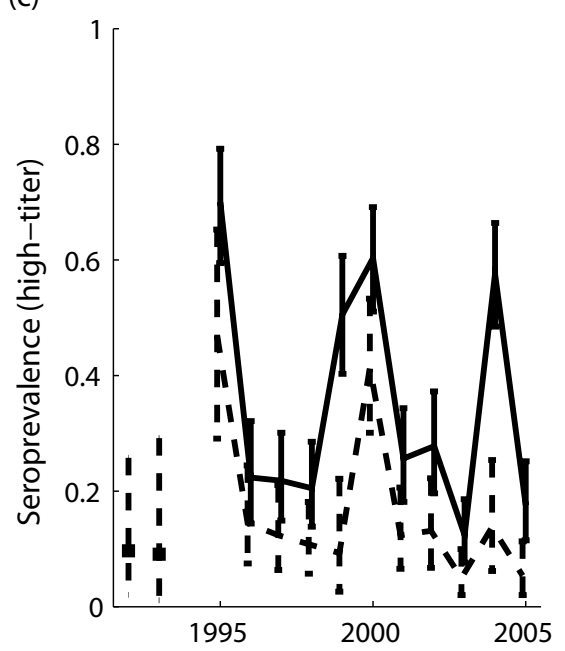

Figure I

Cycles in seroprevalence to $L$. interrogans serovar Pomona in California sea lions stranding in northern California. Three panels correspond to (a) total seroprevalence (titers $\geq 1: 100$ ), (b) low-titer seroprevalence (titers $\geq 1: 100$ and $<1: 800$ ), and (c) high-titer seroprevalence (titers $\geq 1: 800$ ). Solid lines show estimates derived from a random sample of all stranded sea lions, including those judged to have stranded due to leptospirosis. Dashed lines show estimated seroprevalence after leptospirosis strands are removed from the dataset. Isolated points in 1992 and 1993 show estimates derived from nonrandomized samples from an earlier publication (I8). Error bars show $95 \%$ confidence intervals. 
(a)

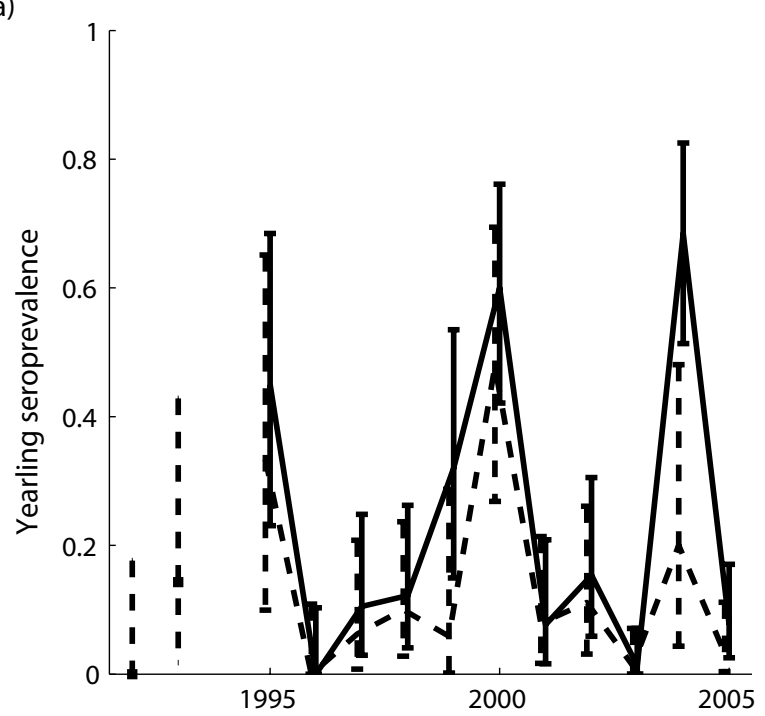

(b)

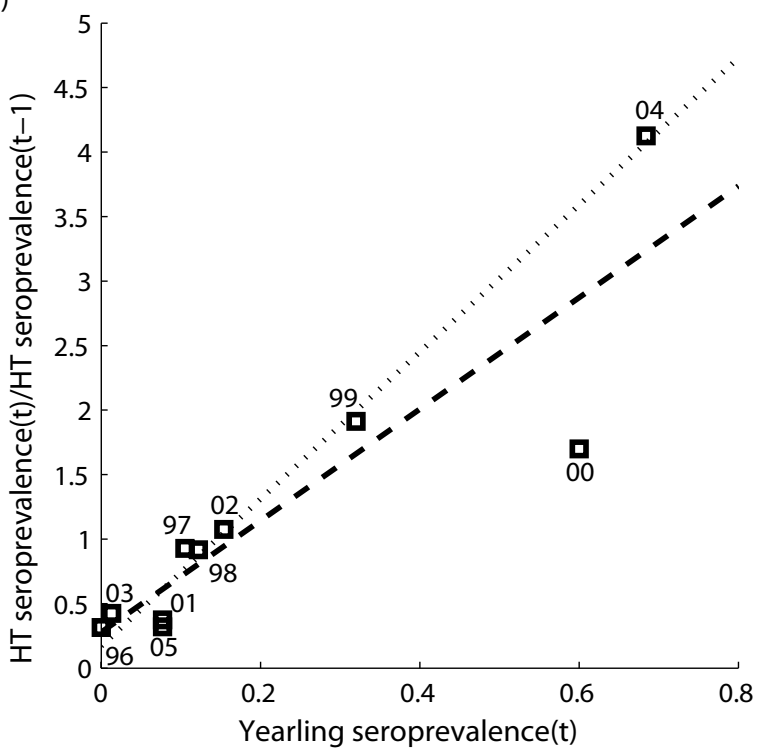

Figure 2

Yearling seroprevalence and relation to exposure. (a) Time series of estimated yearling seroprevalence, estimated as in Figure I. (b) Linear regression of the relative change in high-titer seroprevalence for the whole population against yearling seroprevalence. Squares show data points for particular years (labeled by the final two digits of the calendar year $t$ ). The dashed line shows the regression including all points; the dotted line shows the regression with the point for the year 2000 excluded.

sharply for adults. Note that the juvenile class contains only males (Table 1), so the sex effect may account for some of the elevated risk for the juvenile class. The relative risk of low titers versus seronegativity was not influenced by outbreak years $(p=0.31)$ or season $(p=0.60)$. In sharp contrast to high-titer results, the relative risk of low positive titers increases steadily with age, with subadults and adults respectively at 24 -fold and 44 -fold higher risk than yearlings to have low titers.

Of 514 sea lions with positive titers, 200 stranded for reasons other than leptospirosis; we called these individuals asymptomatic seropositives. The proportion of asymptomatic seropositives was higher among low-titer individuals than high-titer individuals (72/79 versus $128 / 435$, $p<$ $0.001)$. This pattern held for males and females analyzed independently ( $p<0.001$ in each case). The asymptomatic proportion showed a weak tendency to be higher in females than males $(p=0.13)$. A greater proportion of seropositive individuals were asymptomatic in non-outbreak years than outbreak years $(p<0.001)$. The asymptomatic proportion was higher among adults than younger age classes $(p<0.001)$; this pattern held for high titers $(p$ $<0.001)$ but not for low titers $(p=0.73)$. Of the 200 asymptomatic seropositives, 30 stranded due to domoic acid toxicity, 40 due to malnutrition, and the remaining 130 due to varied causes including trauma, cancer, behavioral problems and unrecorded causes; we cannot exclude the possibility that some of these animals stranded due to subclinical leptospirosis.

\section{Antibody decay and loss of seropositivity}

Yearling seroprevalence in non-outbreak years was lower than overall seroprevalence. If yearling seroprevalence is an accurate index of transmission within a year (Figure 2b), then this difference indicates that some older individuals maintain seropositivity for one or more years following exposure. The y-intercepts of the regression lines in Figure $2 \mathrm{~b}$ provide estimates of the proportion of individuals that maintain a high titer from one year to the next without being re-exposed, or conversely the proportion $p_{\text {decay }}$ that lose their high titer. When corrections are applied for deaths and recruitment of new unexposed pups (Equation 1), this proportion is estimated to be $p_{\text {decay }}$ $=0.69(95 \%$ CI $0.06-1)$ for the full dataset, or $p_{\text {decay }}=0.81$ (95\% CI 0.59-1) when the 2000 point is excluded. Interpreting these values requires consideration of the quantitative distribution of titer scores. After stimulation by the antigen has ceased, circulating antibodies are conventionally thought to decay exponentially, with half-life of IgG estimated as 23-25 days [37]. Because titers are scored by two-fold dilutions, a drop in titer on a $\log _{2}$ scale corresponds to the intervening number of half-lives. Of 248 seropositive samples assayed to dilutions up to $1: 204800$, 208 were high-titer $(\geq 1: 800)$ and 142 were positive at the maximum dilution so their precise titer is unknown (Figure 3a). The remaining 66/208 (32\%) of high-titer scores are between 1 and 8 two-fold dilutions from the 1:800 
Table 4: Risk factors for high-titer $(\geq 1: 800)$ and low-titer $(\geq 1: 100$ and $<1: 800)$ seropositivity to $L$. interrogans serovar Pomona. Significant variables in the multinomial logistic regression model and corresponding relative risk ratios (RRR) are shown. ${ }^{a}$

\begin{tabular}{|c|c|c|c|c|c|c|c|c|}
\hline \multirow[b]{2}{*}{ Variable } & \multirow[b]{2}{*}{ Value } & \multirow[b]{2}{*}{$\mathbf{N}^{\mathrm{b}}$} & \multicolumn{3}{|c|}{ High titer seropositivity vs seronegativity } & \multicolumn{3}{|c|}{ Low titer seropositivity vs seronegativity } \\
\hline & & & RRR & $95 \% \mathrm{Cl}$ & p-value & RRR & $95 \% \mathrm{Cl}$ & p-value \\
\hline \multirow[t]{4}{*}{ Age } & Yearling & $450(383)$ & 1 & & & 1 & & \\
\hline & Juvenilec & $220(99)$ & 4.41 & $(2.80,6.95)$ & $<0.001$ & 5.99 & $(1.5,23.9)$ & 0.011 \\
\hline & Subadult & $331(195)$ & 3.79 & $(2.54,5.67)$ & $<0.001$ & 23.8 & $(6.85,82.6)$ & $<0.001$ \\
\hline & Adult & $293(241)$ & 0.79 & $(0.49,1.27)$ & 0.32 & 43.6 & $(12.9,147.4)$ & $<0.001$ \\
\hline \multirow[t]{2}{*}{ Sex } & Female & $565(456)$ & I & & & 1 & & \\
\hline & Male & 729 (462) & 4.13 & $(2.89,5.89)$ & $<0.001$ & 8.29 & $(4.62,14.9)$ & $<0.001$ \\
\hline \multirow[t]{2}{*}{ Season } & Jan-Jul & 629 (549) & 1 & & & 1 & & \\
\hline & Aug-Dec & $665(369)$ & 4.87 & $(3.53,6.72)$ & $<0.001$ & 1.15 & $(0.68,1.92)$ & 0.60 \\
\hline \multirow[t]{2}{*}{ Outbreak year } & No & $859(710)$ & 1 & & & 1 & & \\
\hline & Yes & 435 (208) & 6.97 & $(5.02,9.68)$ & $<0.001$ & 1.35 & $(0.76,2.39)$ & 0.31 \\
\hline
\end{tabular}

a Model had residual deviance 1483.3 on $1287 \mathrm{df}$ (chi-squared $p=\mathrm{I}$; Nagelkerke $R^{2}=0.5 \mathrm{I}$ ).

b Sample size in each group is shown as a total number and as a number excluding leptospirosis-induced strands (in parentheses).

c Note that the juvenile age class contains only males.

threshold for high titers, so the high titer would be lost after 1-8 half-lives with no re-exposure. However, 142/ $208(68 \%)$ of high-titer scores are "off the charts", so we cannot assess how many half-lives they are from the threshold. We can conclude only that the titer distributions predict that $\geq 32 \%$ of high-titer individuals should drop to low-titer or become seronegative after 8 half-lives without exposure. This is consistent with the regression estimates of $69 \%$ and $81 \%$, but does not test the precise values.

Antibody decay is evident in titer distributions during periods of low exposure. Titers from non-outbreak years showed more intermediate values and fewer maximum values than titers from outbreak years (Figure 3b). When stratified by season, titer distributions were heavily skewed toward the highest values during the leptospirosis outbreak seasons of July-September and October-December, then declined progressively to lower values in January-March and April-June (Figure 3c). These patterns cannot be analyzed quantitatively because of seasonal differences in stranding rates across age classes, but the same qualitative patterns arose when yearling or adult samples were excluded from the analysis.

Direct estimates of titer decay rates could be made from six paired serum samples available from California sea lions undergoing rehabilitation following infection. Because the second sample in each instance was seronegative (and we did not know when the detection threshold was crossed), only a minimum bound on decay rate could be estimated from these data. The boundary of the allowable region for decay rates is defined by the best-fit line through the origin and the three fastest-decaying points, which had slope 0.050 (95\% CI $\left.0.046,0.054 ; R^{2}=0.92\right)$ corresponding to a half-life of 20 (19-22) days.

\section{Comparison with animals stranded in southern California}

We compared the seroprevalence of sea lions sampled in southern California to the TMMC time series (Figure 4). The 2000 outbreak was reflected in the high-titer seroprevalence from SeaWorld (though sample size was only $n=$ 4). The 2004 outbreak was not evident at either southern California site: high-titer seroprevalence was greater at TMMC than MMCC $(p=0.002)$ and SeaWorld $(p<$ $0.001)$, as was yearling seroprevalence ( $p=0.047$ and $p<$ 0.001 , respectively).

\section{Discussion}

Leptospirosis in California sea lions does not fit neatly into the classical dichotomous framework wherein a given host species is either a maintenance host or an accidental host for a particular serovar of pathogenic Leptospira. In sea lions the disease exhibits characteristics of accidental hosts, with pathogenic and sometimes fatal outcomes for individual animals and dramatic outbreaks at the population scale. Yet the disease also appears to circulate at low levels between outbreaks, and asymptomatic seropositivity (even with high titers) is common among adults. Other investigators have reported chronic shedding of leptospires, another characteristic of classical maintenance hosts, with one sea lion reported to shed for at least 154 days following infection [17].

All available evidence, including several isolates from wild sea lions $[15,18,28]$ and comparison of MAT titer scores (Table 3), points to L. interrogans serovar Pomona as the cause of leptospirosis in this population. Mixing of hostadapted and non-adapted traits may be a property of serovar Pomona, which causes disease in pigs, cattle and horses, but can also be shed for 4-6 months by those species [38-40]. Of course, the possibility that other serovars are circulating cannot be excluded without intensive 
(a)

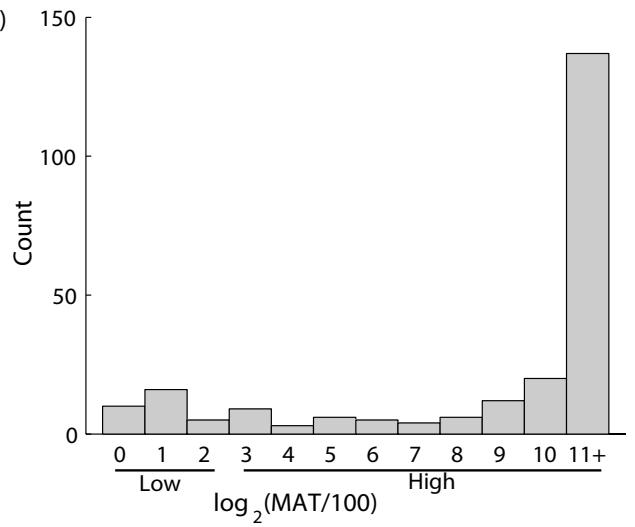

(b)
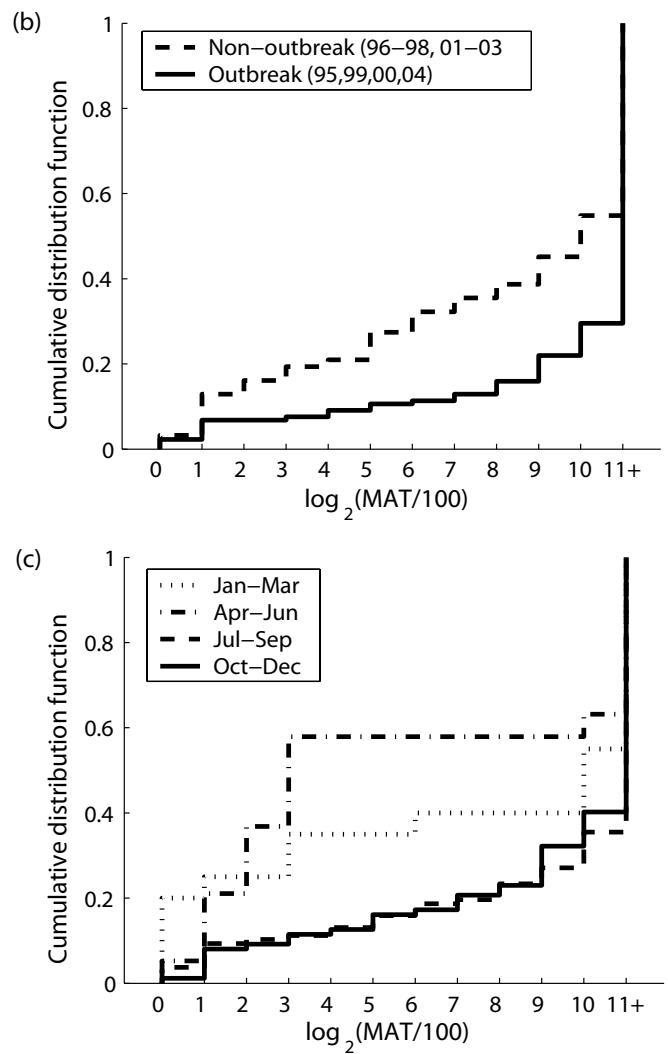

Figure 3

Distributions of MAT scores from the 1995-2005 time series. Part (a) shows the histogram of all 248 positive titers among the 724 samples that were measured to a maximum dilution of $\mathrm{I}: 204800$. Parts (b) and (c) show distributions of different subdivisions of these 248 scores, represented as cumulative distribution functions that show the proportion of samples with scores less than or equal to a given value. Titer scores are represented as $\log _{2}(\mathrm{MAT} / \mathrm{I00})$, such that a score of 0 corresponds to a titer of $\mathrm{I}: \mathrm{I00}$, a score of I to a titer of I:200, etc. The highest score of II corresponds to titers $\geq 1: 204800$. Sample sizes for (c) are Jan-Mar $(N=2 I)$, Apr-Jun $(N=2 I)$, Jul-Sep $(N=I I 5)$ and Oct-Dec $(\mathrm{N}=91)$. efforts to isolate and identify further leptospires from wild sea lions. Two serovars that were not included in our MAT panel warrant special mention.L. interrogans serovar Autumnalis is increasingly reported in serological studies of dogs in the United States [11] and is known to crossreact with Pomona in the MAT $[11,41]$. L. kirschneri serovar Cynopteri has been detected serologically in a geographically separated population of $Z$. californianus in the Gulf of California [42], but the highest titer observed (1:50) was below our threshold for seropositivity. Ongoing circulation of serovar Pomona remains the most parsimonious explanation for the available data.

If the classical maintenance/accidental-host model of leptospirosis epidemiology is overly simplified, then public health officials must broaden their view of potential reservoirs for this zoonotic pathogen. If the California sea lion population is indeed a reservoir for serovar Pomona, then health warnings regarding leptospirosis risk from stranded sea lions should be extended to non-outbreak periods. The possibility that on-going exposure of sea lions to serovar Pomona arises from continuous contact with an unidentified external reservoir cannot be excluded, but this explanation would only broaden the public health implication to include other host species.

The present study identifies clear cycles of 4-5 year periodicity in seroprevalence to $L$. interrogans serovar Pomona in the California sea lion population off the California coast. The cyclic pattern arises from changes in the prevalence of high titer scores reflective of recent exposure, and peak years of the cycle correspond to observed peaks in sea lions stranding with leptospirosis. Seroprevalence in yearling sea lions is strongly correlated with annual changes in high-titer seroprevalence for the whole population, and indicates on-going exposure to serovar Pomona between outbreak years; this finding is consistent with earlier studies reporting continued stranding and death due to leptospirosis in non-outbreak years $[18,19]$, and inconsistent with expected patterns for an accidental host. Evaluation of individual risk factors reveals that juvenile and subadult animals are at greatest risk for hightiter seropositivity, while adults are at sharply reduced risk.

These observations suggest strongly that leptospirosis is endemic within the sea lion population, and raise the intriguing possibility that repeated epidemics arise from the intrinsic interaction of birth rates and herd immunity, rather than the environmental drivers that are commonly postulated. Younger animals get infected in outbreak years, acquiring high titers that may persist for a year or more, while most adults are immune from previous exposure. The pathogen may persist through off-seasons and non-outbreak years via chronic infections and a low level 
(a)

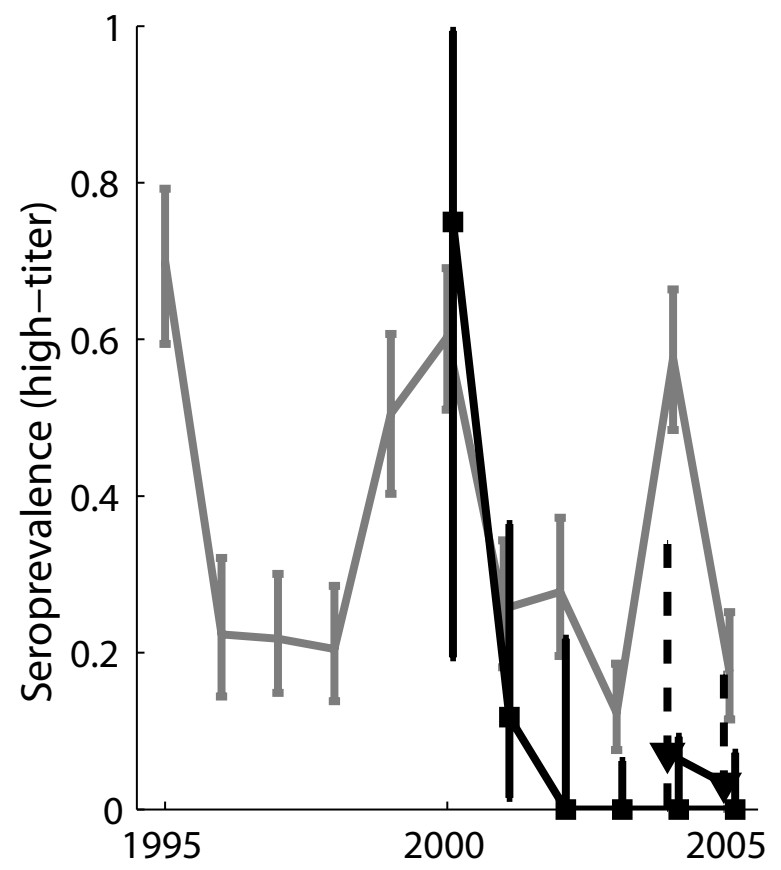

(b)

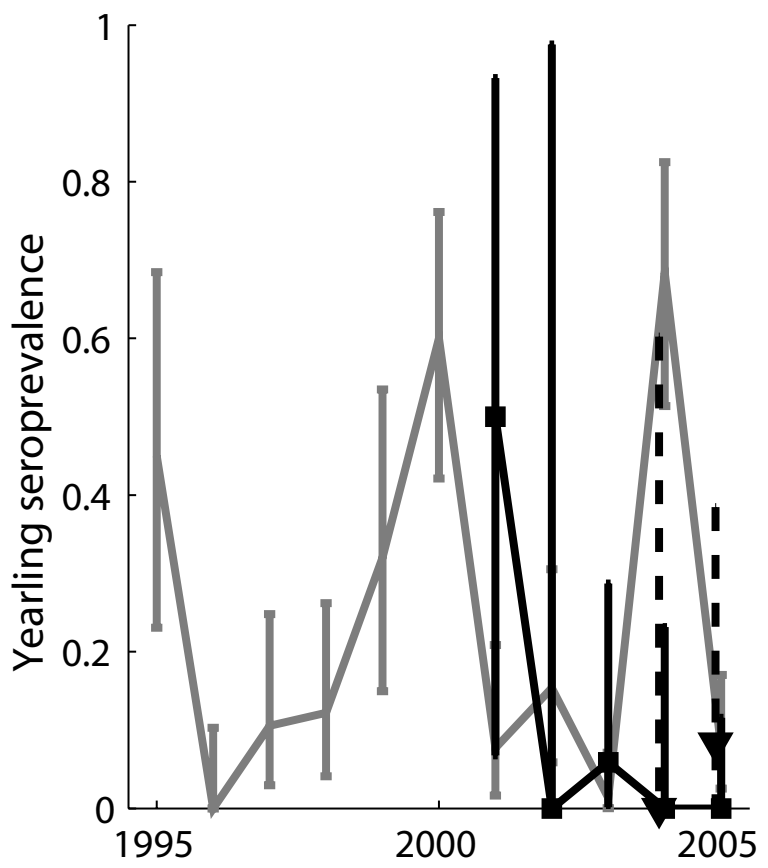

Figure 4

Seroprevalence in stranded California sea lions: other datasets. High-titer and yearling seroprevalence from other stranding ranges in southern California. For comparison, estimates from the 1995-2005 time series are shown in gray (full dataset, including leptospirosis-induced strands). Solid lines and squares show estimates from SeaWorld (San Diego CA), while dashed lines and triangles show estimates from the Marine Mammal Care Center (Fort Macarthur CA).

of on-going transmission, possibly associated with the increased prevalence of asymptomatic seropositivity and low titers during these periods. We emphasize, though, that present data cannot exclude the possibility of ongoing contact with another reservoir species. Mathematical models integrating the dynamics of disease transmission and immunity with sea lion demographics, combined with further data collection, are essential to clarify this issue.

Live-stranded marine mammals are a biased sample of the wild population, over-representing sick and weak individuals, so seroprevalence in stranded individuals may exceed the true population value. We addressed this bias by providing alternate seroprevalence estimates based on a reduced dataset excluding clinical leptospirosis cases, but this approach may yield underestimates during outbreaks if a substantial proportion of infected animals do not come ashore. Of over 200,000 sea lions breeding off the California coast [34], just a few hundred leptospirosis cases strand and are admitted to rehabilitation during a typical outbreak year [19]. Given peak seroprevalence estimates $>50 \%$, it appears that many infected animals do not strand. Serosurveillance of free-ranging sea lions is crucial to determine how the patterns reported here scale to the population level.

Data from sea lions stranded in southern California showed a general trend of lower seroprevalence than was found in central and northern California, and, intriguingly, there is no evidence of the 2004 outbreak in data from the southern range. All California sea lions in the eastern Pacific Ocean breed on rookery islands off the coast of southern California and the Baja peninsula, so animals stranding in different regions of California are thought to be drawn from a single population. Leptospirosis strandings (and high-titer seropositivity) peak during July to November [19], when sea lions migrate northward following the breeding season to forage off central and northern California, or points further north. It is unknown whether this timing is coincidental or leptospirosis transmission (or exposure) is aided by environmental factors in the northern range. Male sea lions migrate further north than females, and in greater numbers, while breeding females remain closer to the rookeries to nurse pups. This difference in migratory behavior 
may play a role in the observed sex difference in leptospirosis incidence, although similar sex differences have been observed in other species including humans and are not easily explained $[13,43]$. Data presented here suggest that leptospirosis incidence is lower among animals remaining in southern California, but increased sampling of stranded and wild-caught individuals is needed to confirm this pattern.

The predominance of high titers among seropositive sea lions (also reported by Colagross-Schouten [27]) indicates that most animals had been exposed recently before stranding. Yet the time course of titer decay for this hostserovar interaction is unknown, so we assembled evidence to assess the duration of high MAT titers in sea lions. Population-level changes in seroprevalence suggest that approximately $69 \%$ of high-titer individuals lose their high titer in a year without re-exposure to the pathogen (but note the broad 95\% CI, 0.06-100\%). This is consistent with observed titer distributions in sea lions, which indicate that $\geq 32 \%$ of individuals should lose their high titers after 8 antibody half-lives, but more precise predictions cannot be derived from titer distributions because the majority of sea lion titers were positive at the highest dilution measured. Maintenance of high MAT titers for several years is not reported in experimental infection studies, which rarely last that long, but has been reported for humans following severe infections [1,21-23]. MAT titer half-life for sea lions in rehabilitation was estimated crudely to have upper bound 20 (19-22) days, lower than the conventional half-life of 23-25 days for IgG antibodies. While this difference could be attributed to numerous factors, including that MAT titers reflect both IgM and IgG levels [1] and antibiotic therapy may reduce titer duration [35], it is important to note the small sample size underlying the estimate, and the well-established finding that human MAT decay rates vary substantially [21-23]. Titer distributions in stranded sea lions are qualitatively consistent with gradual antibody decay following exposure, but intermediate titers (from 1:3200 to 1:51200) appear less commonly than a simple exponential decay model would predict. Longitudinal titers from individuals recovering from acute infection are required to characterize the true rate of antibody decay from high levels. The possible role of chronic shedders, in maintaining their own lowtiter seropositivity and in boosting the antibody responses of others, requires investigation.

\section{Conclusion}

This study presents results of an unprecedented serosurveillance program in marine mammals, tracking seroprevalence to L. interrogans serovar Pomona in 1344 stranded California sea lions over 11 years. The data show cycles of 4-5 year periodicity, with peaks corresponding to observed increases in sea lion strandings due to lept- ospirosis. Seroprevalence in yearling sea lions is an accurate indicator of incidence of leptospiral infection for all age classes, and indicates on-going exposure to serovar Pomona between outbreak years. These results suggest that sea lions occupy a middle ground between classicallydefined maintenance and accidental hosts of leptospirosis, but many questions remain. Further data are needed to address crucial uncertainties such as the seroprevalence of the free-ranging population, the prevalence of chronic shedding among sea lions, and the spatiotemporal interaction of sea lion migration and leptospirosis risk. Molecular comparisons of $L$. interrogans isolates from sea lions and possible external reservoirs would contribute vital evidence regarding the question of endemic persistence versus repeated introductions. Further analysis of existing data is also essential, particularly via mathematical models that integrate the dynamics of disease transmission, population growth, and antibody decay with all available data types. The sea lion/leptospirosis system raises questions regarding the accepted view of the epidemiology of this important zoonosis, but only through a sustained interdisciplinary effort will definitive answers be obtained.

\section{Competing interests}

The author(s) declare that they have no competing interests.

\section{Authors' contributions}

JL-S analyzed the data and drafted the manuscript. DG managed the samples and primary data at TMMC, and helped to interpret the results and draft the manuscript. GG coordinated the early phase of the data collection. SH coordinated the serological analysis and advised on its interpretation. LP and JSL contributed samples and accompanying data from southern California. BG advised on the analysis and interpretation of the time series, and helped to draft the manuscript. FG conceived of the study, participated in its design and coordination, and helped to interpret the results and draft the manuscript. All authors read and approved the final manuscript.

\section{Acknowledgements}

We thank the staff at The Marine Mammal Center (TMMC), the Marine Mammal Care Center at Fort Macarthur, and SeaWorld for assisting in the collection of these data, and the staff at the California Animal Health and Food Safety Laboratory for conducting the microscopic agglutination tests. We are grateful to Carole Bolin for insightful discussion of leptospirosis serology.

This research was supported by the National Marine Fisheries Service Marine Mammal Health and Stranding Research Program. JLS was supported by a Center for Infectious Disease Dynamics Fellowship from Penn State University; DG and FG were supported by the Oceans and Human Health Initiative. 


\section{References}

I. Faine S, Adler B, Bolin C, Perolat P: Leptospira and leptospirosis 2nd edition. Melbourne, Australia: MediSci; 1999.

2. Levett PN: Leptospirosis. Clin Microbiol Rev 200I, I4:296-326.

3. Leighton FA, Kuiken T: Leptospirosis. In Infectious diseases of wild mammals Edited by: Williams ES, Barker IK. Ames: lowa State University Press; 2001:498-50I

4. Bharti AR, Nally JE, Ricaldi JN, Matthias MA, Diaz MM, Lovett MA Levett PN, Gilman RH, Willig MR, Gotuzzo E, Vinetz JM: Leptospirosis: a zoonotic disease of global importance. Lancet Infectious Diseases 2003, 3:757-77I.

5. McBride AJA, Athanazio DA, Reis MG, Ko Al: Leptospirosis. Cur Opin Infect Dis 2005, I 8:376-386.

6. Trevejo RT, Rigau-Perez JG, Ashford DA, McClure EM, JarquinGonzalez C, Amador JJ, de los Reyes JO, Gonzalez A, Zaki SR, Shieh W], et al.: Epidemic leptospirosis associated with pulmonary hemorrhage - Nicaragua, 1995. J Infect Dis 1998 I 78: | 457-| 463 .

7. Romero EC, Yasuda PH: Molecular characterization of Leptospira sp strains isolated from human subjects in Sao Paulo, Brazil using a polymerase chain reaction-based assay: a public health tool. Mem Inst Oswaldo Cruz 2006, I 0 I:373-378.

8. Jena $A B$, Mohanty KC, Devadasan N: An outbreak of leptospirosis in Orissa, India: the importance of surveillance. Trop Med Int Health 2004, 9:1016-1021.

9. Laras K, Van CB, Bounlu K, Tien NTK, Olson JG, Thongchanh S, Van Anh TN, Loan HK, Punjabi N, Khiem HB, et al: The importance of leptospirosis in Southeast Asia. Am J Trop Med Hyg 2002 67:278-286.

10. Bolin CA: Diagnosis of leptospirosis: A reemerging disease of companion animals. Semin Vet Med Surg (Small Anim) 1996, I I:I66-17|

I I. Moore GE, Guptill LF, Glickman NW, Caldanaro RJ, Aucoin D, Glickman LT: Canine leptospirosis, United States, 2002-2004. Emerg Infect Dis 2006, I 2:501-503.

12. Higgins R: Emerging or re-emerging bacterial zoonotic diseases: bartonellosis, leptospirosis, Lyme borreliosis, plague. Rev Sci Tech 2004, 23:569-58I.

13. Adin CA, Cowgill LD: Treatment and outcome of dogs with leptospirosis: 36 cases (1990-1998). J Am Vet Med Assoc 2000 216:37|-375.

14. Meites E, Jay MT, Deresinski S, Shieh WJ, Zaki SR, Tompkins L, Smith DS: Reemerging leptospirosis, California. Emerg Infect Dis 2004 10:406-4I2

I5. Mcllhattan TJ, Martin JW, Wagner RJ, Iverson JO: Isolation of Leptospira pomona from a naturally infected California sea lion, Sonoma County, California. J Wildl Dis 197I, 7:195-197.

16. Vedros NA, Smith AW, Schonewa J, Migaki G, Hubbard RC: Leptospirosis epizootic among California sea lions. Science 197I, I72: |250-|25|.

17. Dierauf LA, Vandenbroek DJ, Roletto J, Koski M, Amaya L, Gage LJ: An epizootic of leptospirosis in California sea lions. J Am Vet Med Assoc 1985, I 87: I | 45- I | 48.

18. Gulland FMD, Koski M, Lowenstine LJ, Colagross A, Morgan L, Spraker T: Leptospirosis in California sea lions (Zalophus californianus) stranded along the central California coast, | 98 |-| 994. J Wild Dis 1996, 32:572-580.

19. Greig DJ, Gulland FMD, Kreuder C: A decade of live California sea lion (Zalophus californianus) strandings along the central California coast: causes and trends, 1991-2000. Aquatic Mammals 2005, 3 I: I I-22

20. Grenfell BT, Dobson AP, Eds: Ecology of Infectious Diseases in Natural Populations. Cambridge: Cambridge University Press 1995.

21. Blackmore DK, Schollum LM, Moriarty KM: The magnitude and duration of titers of leptospiral agglutinins in human sera. $N$ Z Med J 1984, 97:83-86.

22. Lupidi R, Cinco M, Balanzin D, Delprete E, Varaldo PE: Serological follow-up of patients involved in a localized outbreak of leptospirosis. J Clin Microbiol I99I, 29:805-809.

23. Cumberland P, Everard COR, Wheeler JG, Levett PN: Persistence of anti-leptospiral IgM, IgG and agglutinating antibodies in patients presenting with acute febrile illness in Barbados 1979-I 989. Eur J Epidemiol 200 I, I 7:601-608.
24. Cole JR Jr, Sulzer CR, Pursell AR: Improved microtechnique for the leptospiral microscopic agglutination test. Appl Microbiol 1973, 25(6):976-980

25. National Veterinary Services Laboratory: Microtiter technique for detection of leptospiral antibodies. United States Animal Health Association 1987:65-73.

26. World Organization for Animal Health: Manual of Diagnostic Tests and Vaccines for Terrestrial Animals 5th edition. 2004.

27. Colagross-Schouten AM, Mazet JAK, Gulland FMD, Miller MA, Hietala $S$ : Diagnosis and seroprevalence of leptospirosis in California sea lions from coastal California. J Wildl Dis 2002, 38:7-I7.

28. Smith AW, Vedros NA, Gilmartin G, Akers TG: Hazards of disease transfer from marine mammals to land mammals - review and recent findings. J Am Vet Med Assoc 1978, I 73: I I3 I-I I33.

29. R Development Core Team: R: A Language and Environment for Statistical Computing. Book R: A Language and Environment for Statistical Computing, City 2.2.I edition. 2006.

30. Clopper C], Pearson ES: The use of confidence or fiducial limits illustrated in the case of the binomial. Biometrika 1934 , 26:404-4I3

3I. Christensen R: Log-Linear Models and Logistic Regression 2nd edition. Berlin: Springer; 1997

32. Tabachnick BG, Fidell LS: Using Multivariate Statistics 5 th edition. Boston: Pearson; 2007.

33. Rice JA: Mathematical statistics and data analysis 2nd edition. Belmont CA: Duxbury Press; 1995.

34. Carretta JV, Forney KA, Muto MM, Barlow J, Baker J, Hanson B, Lowry MS: U. S. Pacific marine mammal stock assessments: 2005. Book U. S. Pacific marine mammal stock assessments: 2005, City 2006.

35. Harkin KR, Gartrell CL: Canine leptospirosis in New Jersey and Michigan: I7 cases (1990-1995). J Am Anim Hosp Assoc 1996, 32:495-50I

36. Melin SR: The foraging ecology and reproduction of the California sea lion (Zalophus californianus californianus). University of Minnesota 2002.

37. Hathaway SC, Little TWA, Jones TWH, Stevens H, Butland RW: Infection by leptospires of the Pomona serogroup in cattle and pigs in south west England. Vet $\operatorname{Rec} 1984$, I I 5:246-248.

38. York CJ, Baker JA: Vaccination for bovine leptospirosis. Am J Vet Res 1953, 14:5-8.

39. Morter RL, Herschler RC. Fessler JF, Lavignette A: Experimental equine leptospirosis (Leptospira pomona). Proc Annu Meet U S Anim Health Assoc 1964, 68: 147-152.

40. Hunter P: Leptospirosis. In Infectious diseases of livestock Volume 3 3rd edition. Edited by: Coetzer JAW, Tustin RC. Oxford: Oxford University Press; 1994: | 445-| 456.

4I. Prescott JF, McEwen B, Taylor J, Woods JP, Abrams-Ogg A, Wilcock $B$ : Resurgence of leptospirosis in dogs in Ontario: recent findings. Can Vet J 2002, 43:955-96I.

42. Acevedo-Whitehouse $\mathrm{K}$, de la Cueva H, Gulland FMD, AuriolesGamboa D, Arellano-Carbajal F, Suarez-Guemes F: Evidence of Leptospira interrogans infection in California sea lion pups from the Gulf of California. I Wild Dis 2003, 39: |45- I5 I.

43. Romero EC, Bernardo CCM, Yasuda PH: Human leptospirosis: a twenty-nine year serological study in Sao Paulo, Brazil. Rev Inst Med Trop Sao Paulo 2003, 45:245-248.

\section{Pre-publication history}

The pre-publication history for this paper can be accessed here:

\section{http://www.biomedcentral.com/1471-2334/7/125/pre} pub 